\title{
Electron mobility in quasi-one-dimensional conductors: A theoretical study
}

\author{
C. E. Leal, ${ }^{*}$ I. C. da Cunha Lima, and E. A. de Andrada e Silva \\ Instituto de Pesquisas Espaciais, Avenida dos Astronautas 1758, 12200 São José dos Campos, São Paulo, Brazil \\ A. Troper \\ Centro Brasileiro de Pesquisas Físicas, rua Dr. Xavier Sigaud 150, 22290 Rio de Janeiro, Rio de Janeiro, Brazil
}

(Received 13 November 1987; revised manuscript received 7 March 1988)

\begin{abstract}
We present a calculation of the temperature dependence of the electronic mobility in quasi-onedimensional conductor systems due to scattering by longitudinal-acoustic phonons, using the memory-function formalism. We expect that the theoretical results here obtained can be applied to quantum-well wires, to linear chain mercury compounds such as $\mathrm{Hg}_{3-\delta} \mathrm{AsF}_{6}$, and to some organic compounds that behave like one-dimensional electron-gas systems.
\end{abstract}

\section{INTRODUCTION}

In recent years much attention has been focused on one-dimensional (1D) metals, mainly due to the fact that some 1D conductor materials present a peculiar instability called the Peierls transition. A gap opens at the Fermi level and a sharp maximum appears in $\partial \ln \rho / \partial T$ at the transition temperature, where $\rho$ is the resistivity of the system. This decrease in the conductivity and the lattice modulation was explained, at first, by the Peierls mechanism, ${ }^{1}$ based on the electron-phonon (EP) interaction, which leads to the appearance of a charge-density wave (CDW) and an associated lattice modulation of wavelength $\pi / k_{F}$ (where $k_{F}$ is the Fermi-surface wave vector) in the one-dimensional electron gas (1D EG). This CDW state has been seen in many 1D metallic materials, e.g., the organic conductor tetrathiafulvalene-tetracyanoquinodimethane $^{2}$ (TTF-TCNQ). However, several materials seem to be $1 \mathrm{D}$ metals and yet do not exhibit a Peierls transition. For instance, $\mathrm{Hg}_{3-\delta} \mathrm{AsF}_{6}$ is an inorganic compound where the incommensurate linear $\mathrm{Hg}$ chain structure in the $\mathrm{AsF}_{6}$ host leads to one-dimensional lattice dynamics and to highly anisotropic electronic properties. $^{3}$ Other examples of quasi-one-dimensional systems are the organic compounds (TMTSF) ${ }_{2} \mathrm{PF}_{6}$ and (TMTSF) ${ }_{2} \mathrm{AsF}_{6}$, which consist of planar stacks of TMTSF molecules with $\mathrm{PF}_{6}$ or $\mathrm{AsF}_{6}$ complexes stacked separately. ${ }^{4}$

On the other hand, some interest has recently arisen in understanding the electronic transport properties of the $1 \mathrm{D} \mathrm{GaAs} / \mathrm{Ga}_{1-x} \mathrm{Al}_{x}$ As quantum-well-wire (QWW) system, which consists of an electron gas tightly confined in ultrafine semiconductor wire structures. ${ }^{5,6}$ A calculation of $1 \mathrm{D}$ mobility limited by ionized donors has been reported for zero temperature by Sakaki ${ }^{5}$ and several works ${ }^{7,8}$ have also appeared in the literature, thus illustrating an increasing interest in these 1D systems.

In this work we are interested in examining more closely this kind of $1 \mathrm{D}$ material with a quantum-wire-like structure that do not have a Peierls instability. We use the memory-function (MF) projection-operator formalism to describe the temperature dependence of their electron- ic transport properties. As shown in a previous work, ${ }^{9}$ this technique becomes very convenient in the case of longitudinal-acoustic (LA) phonon scattering systems for both high- and low-temperature regimes. Besides, it allows one to include the presence of high magnetic fields without further formal difficulties. In Sec. II, we exhibit the Hamiltonian model of the problem, as well as the MF formalism, in order to derive a formal expression for the conductivity through the calculation of the $1 \mathrm{DEG}$ dielectric function. This section is also devoted to the numerical analysis of the temperature behavior of the electrical mobility. Finally, in Sec. III we summarize the main conclusions and make supplementary comments concerning systems that also exhibit $1 \mathrm{DEG}$ character.

\section{THE MODEL HAMILTONIAN, MF FORMALISM, AND NUMERICAL RESULTS}

Since the number of thermally excited optical phonons in the temperature range of our interest is quite small, it is not expected that they will play a fundamental role in the scattering process of the 1D EG. Therefore, we shall consider in this work only the acoustic-phonon scattering, using the deformation-potential method at longwavelength limit. In this way, we use the Fröhlich Hamiltonian describing the interaction between a onedimensional electron gas and a 1D LA acoustic phonon as

$$
H=\sum_{\mathbf{k}} \epsilon_{\mathrm{k}} C_{\mathbf{k}}^{\dagger} C_{\mathbf{k}}+\sum_{\mathbf{q}} \hbar \omega_{\mathbf{q}} a_{\mathrm{q}}^{\dagger} a_{\mathbf{q}}+\sum_{\mathbf{q}} D(\mathbf{q}) \rho(\mathbf{q}) A_{\mathbf{q}},
$$

with

$$
\begin{aligned}
& D(\mathbf{q})=\left[\left(\hbar / 2 m_{i} N^{\prime} \omega_{\mathrm{q}}\right)\right]^{1 / 2} q \xi, \\
& \rho(\mathbf{q})=\sum_{\mathbf{k}} C_{\mathrm{k}+\mathrm{q}}^{\dagger} C_{\mathrm{k}},
\end{aligned}
$$

and

$$
A_{\mathrm{q}}=\left(a_{\mathrm{q}}+a_{-\mathrm{q}}^{\dagger}\right) \text {. }
$$

Here $C_{\mathrm{k}}^{\dagger}\left(C_{\mathrm{k}}\right)$ and $a_{\mathrm{q}}^{\dagger}\left(a_{\mathrm{q}}\right)$ are the electron and phonon creation (annihilation) operators with momentum $\mathbf{k}$ and 
q, respectively. The third term in Eq. (1) is the electron-LA-phonon interaction potential where $\rho(q)$ is the Fourier transform of the electron-density operator and $\xi$ is the deformation constant due to lattice dilation. $N^{\prime}$ is the number of lattice cells and $m_{i}$ is the ionic mass. The LA-phonon frequency is represented by the Debye model, i.e., $\omega_{\mathrm{q}}=C_{s} q, C_{s}$ being the velocity of sound and $x$ the unique electron (phonon) propagation coordinate. Since we assume a 1D model for the phonon system, only phonons with wave vectors along the $x$ direction contribute to the scattering and hence to the resistivity.

Then, the conductivity tensor of Kubo's expression can be expressed as

$$
\sigma_{x x}(\omega)=\frac{i}{\omega} \frac{N e^{2}}{m^{*}}+\frac{i}{\omega} \int_{-\infty}^{\infty} d t Q_{x x}(t) e^{i \omega t},
$$

where $m^{*}$ is the electron effective mass $\left(m^{*}=0.067\right.$ a.u., in GaAs), $N$ is the number of carriers with charge $|e|$, and $Q_{x x}(t)$ is the retarded Green function associated with the current-current correlation function $J(t)$ :

$$
Q_{x x}(t)=-i \Theta(t)\left\langle\left[J_{x}(t), J_{x}(0)\right]\right\rangle,
$$

where $\Theta(t)$ is the usual Heaviside step function. [ $\Theta(t)=1$ if $t>0$ and $\Theta(t)=0$ if $t<0$.] For simplicity, we shall omit the subscript $x$ throughout this work.

The dynamical conductivity $\sigma(z)$ can be expressed in terms of a memory function $M(z){ }^{10}$ Let us introduce the spectral function $X(z)$, given by

$X(z)=\frac{1}{\pi} \int_{-\infty}^{\infty} d \omega X^{\prime \prime}(\omega) /(\omega-z)=i \int_{0}^{\infty} d t e^{i z t} X^{\prime \prime}(t)$,

where the spectral function $X^{\prime \prime}(t)$ can be expressed in terms of the current-current correlation function

$$
X^{\prime \prime}(t)=\frac{m^{* 2}}{e^{2}}\langle[J(t), J(0)]\rangle_{\mathrm{eq}} .
$$

After some manipulations, $\sigma(z)$ can be written

$$
\sigma(z)=i \frac{N e^{2}}{m^{*} z}-\frac{i e^{2}}{m^{* 2} z} X(z) \text {. }
$$

The Laplace transform $C(z)$ of the correlation function $C(t)=\left(m^{* 2} / e^{2}\right)\langle J(t) / J(0)\rangle$, can be related to $X(z)$ as

$$
C(z)=-i \frac{\beta^{-1}}{z}[X(z)-X(0)], \quad \beta=\left(k_{B} T\right)^{-1}
$$

where $X(0)$ is the static limit of $X(z)$. Assuming the boundary condition that the system is a normal conductor, i.e., the conductivity tends to a finite value as $z$ approaches zero, we get from Eqs. (9) and (10) the value

$$
X(0)=N m^{*} \text {. }
$$

Then, $C(z)$ obeys the following memory-function equation:

$$
C(z)=i \beta^{-1} X(0) /[z+M(z)] .
$$

The dc electrical conductivity can be obtained in terms of the memory function as
$\sigma^{-1}(0)=\frac{m^{*}}{N e^{2}} \operatorname{Im} M(0), \quad M\left(\omega \pm i 0^{+}\right)=M_{1}(\omega) \pm i M_{2}(\omega)$

In the zeroth-order approximation the MF can be expressed in terms of retarded force-force correlation function $\Pi^{R}(\omega)$,

$$
M^{(0)}(0)=-\frac{1}{N m^{*}} \lim _{\omega \rightarrow 0} \Pi^{R}(\omega) \omega^{-1},
$$

where

$$
\Pi^{R}(\omega)=-i \theta(t) \int_{-\infty}^{\infty}\left\langle\left[U^{\prime}(t), U^{\prime}(0)\right]\right\rangle e^{i \omega t} d t,
$$

$U^{\prime}$ being the generalized force along the $x$ direction:

$$
U^{\prime}=i \sum_{\mathbf{q}} q D(\mathbf{q}) \rho(\mathbf{q}) A_{\mathbf{q}} e^{i q x} .
$$

The retarded force-force correlation function can be written in terms of the electron-density correlation function and phonon propagator: ${ }^{11}$

$\Pi^{R}(i \omega)=-\sum_{\mathbf{q}} q^{2}|D(\mathbf{q})|^{2} \frac{1}{\beta} \sum_{i p_{n}} S\left(\mathbf{q}, i p_{n}\right) D\left(\mathbf{q}, i \omega-i p_{n}\right)$.

We use instead, in zeroth order, $S^{0}\left(\mathbf{q}, i p_{n}\right)$ and $D^{0}\left(\mathbf{q}, i \omega-i p_{n}\right)$, respectively, the noninteracting electron density and free-phonon propagator, defined as

$S^{0}\left(\mathbf{q}, i p_{n}\right)=\frac{1}{\beta} \sum_{\mathbf{k}, \sigma} \sum_{i k_{n}} g^{0}\left(\mathbf{k}+\mathbf{q}, \sigma, i k_{n}-i p_{n}\right) g^{0}\left(\mathbf{k}, \sigma, i k_{n}\right)$,

$D^{0}\left(\mathbf{q}, i \omega-i p_{n}\right)=\frac{-2 \omega_{\mathbf{q}}}{\left(\omega-p_{n}\right)^{2}+\omega_{\mathbf{q}}^{2}}$

After some algebra, using the finite-temperature formalism in the Matsubara representation, we can express the imaginary part of $\Pi^{R}(\omega)$ in terms of the imaginary part of the dielectric constant $\epsilon_{2}(q, \hbar \omega)$ in the randomphase approximation (RPA):

$$
\begin{aligned}
\operatorname{Im}\left[\boldsymbol{I}^{R}(\omega)\right]=-\sum_{\mathbf{q}} q^{2} \mid & \left.D(\mathbf{q})\right|^{2} \\
\times & \left(\frac{e^{\hbar \beta \omega_{\mathbf{q}}}\left(e^{\hbar \beta \omega}-1\right)}{\left(e^{\hbar \beta \omega_{\mathbf{q}}}-1\right)\left(e^{\hbar \beta\left(\omega_{\mathbf{q}}+\omega\right)}-1\right)}\right. \\
& \times \frac{\epsilon_{2}\left(\mathbf{q}, \hbar \omega_{\mathbf{q}}+\hbar \omega\right)}{V_{\mathbf{q}}} \\
& -\mathcal{T}(\omega \rightarrow-\omega))
\end{aligned}
$$

where $V_{\mathrm{q}}$ is the Fourier transform of the Coulomb potential and $\mathcal{T}$ represents the term preceding $\omega$ replaced by $-\omega$. According to Eq. (14), in the static limit $\omega \rightarrow 0$, the imaginary part of the MF is given by

$M_{2}(0)=\frac{2 \hbar \beta}{N m^{*}} \sum_{\mathbf{q}} q^{2}|D(\mathbf{q})|^{2} \frac{e^{\hbar \beta \omega}}{\left(e^{\hbar \beta \omega}-1\right)^{2}} \frac{\epsilon_{2}\left(\mathbf{q}, \hbar \omega_{\mathbf{q}}\right)}{V_{\mathbf{q}}}$. 
The 1D dielectric function calculation turns out to be

$\frac{\epsilon_{2}(\mathbf{q}, \hbar \omega)}{V_{\mathbf{q}}}=\frac{\left(2 m^{*}\right)^{1 / 2}}{\hbar} \epsilon_{\mathbf{q}}^{-1 / 2}\left[f\left(E_{-}\right)-f\left(E_{+}\right)\right]$,

$f(E)$ being the Fermi-Dirac distribution function, $\epsilon_{\mathrm{q}}$ and $E_{\mp}$ being, respectively,

$$
\epsilon_{\mathrm{q}}=\frac{\hbar^{2} q^{2}}{2 m^{*}}
$$

and

$$
E_{\mp}=\frac{\left(\epsilon_{\mathrm{q}} \mp \hbar \omega_{\mathrm{q}}\right)^{2}}{4 \epsilon_{\mathrm{q}}}=\epsilon_{0}\left[\frac{\hbar q}{2 m^{*} C_{s}} \mp 1\right]^{2}
$$

with

$$
\epsilon_{0}=\frac{m^{*} C_{s}^{2}}{2}
$$

So, considering only scattering by $1 \mathrm{D}$ phonons, with a maximum momentum transfer $\left(q_{m}=2 k_{F}\right)$ one has

$$
M_{2}(0)=\frac{\xi^{2} \beta}{\pi N N^{\prime} m_{i} C_{s}}\left(I_{-}-I_{+}\right)
$$

where

$$
I_{\mp}(\beta)=\int_{0}^{2 k_{F}} d q q^{2} \frac{e^{\hbar \beta C_{s} q}}{\left(e^{\hbar \beta C_{s} q}-1\right)^{2}} f\left(E_{\mp}\right) .
$$

Figure 1 shows the behavior of the energies $E_{\mp}(q)$ as a function of the momentum transfer $q$. Performing a $q \rightarrow E$ variable change in each integral equation given by Eq. (27) we have in general form

$$
I(\beta)=\int_{a}^{b} d E g(E) f(E),
$$

which has a simple solution given by taking the leading term in a Sommerfeld expansion: ${ }^{12}$

$$
\begin{aligned}
I(\beta)= & {\left[G\left(E_{F}\right)-G(a)\right] \Theta\left(E_{F}-a\right) } \\
& -\left[G\left(E_{F}\right)-G(b)\right] \Theta\left(E_{F}-b\right),
\end{aligned}
$$

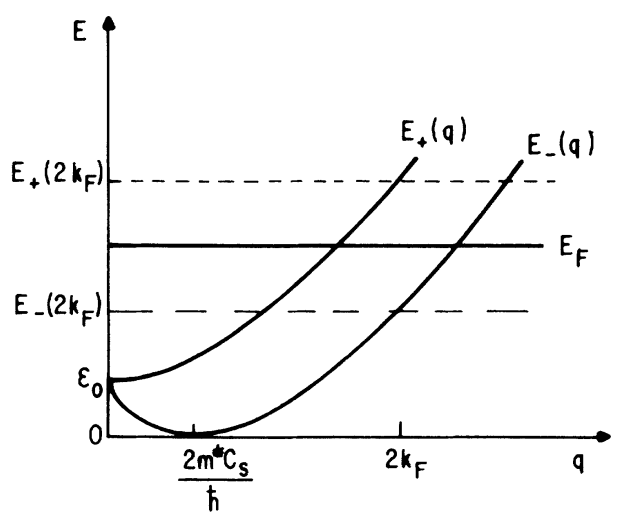

FIG. 1. Energies $E_{+}(q)$ and $E_{-}(q)$ as a function of the momentum transfer $q$. According to Eq. (24) one has $E_{-}\left(2 k_{F}\right)<E_{F}<E_{+}\left(2 k_{F}\right)$. where

$$
G(E)=\int_{0}^{E} g\left(E^{\prime}\right) d E^{\prime} .
$$

Using this method, after some change in variables, we obtain a formal expression to the dc conductivity as a function of the temperature [cf. Eq. (13)]:

$$
\begin{aligned}
\sigma^{-1}(T)= & \frac{m^{*} \xi^{2}\left(k_{B} \Theta_{m}\right)^{2}}{\pi N^{2} e^{2} N^{\prime} m_{i} \hbar^{3} C_{s}^{4}}\left[\frac{T}{\Theta_{m}}\right]^{2} \\
\times & \left\{J_{2}\left[4 \beta \epsilon_{0}\left[1+\frac{\left(E_{F}\right)^{1 / 2}}{\epsilon_{0}}\right]\right]\right. \\
& \left.\quad-J_{2}\left[4 \beta \epsilon_{0}\left[-1+\frac{\left(E_{F}\right)^{1 / 2}}{\epsilon_{0}}\right]\right]\right\} .
\end{aligned}
$$

The temperature $\Theta_{m}$ can be associated to the maximum momentum transfer at the low-temperature regime, i.e., $k_{B} T \ll E_{F}$. For a $\mathrm{GaAs} / \mathrm{Ga}_{1-x} \mathrm{Al}_{x} \mathrm{As}$ quantum wire, one has $\Theta_{m}=4.4 \mathrm{~K}$ whereas $J_{n}(y)$ is the Debye integral:

$$
J_{n}(y)=\int_{0}^{y} d x x^{n} \frac{e^{x}}{\left(e^{x}-1\right)^{2}} .
$$

Finally, the static mobility $\mu(T)$, obtained directly from Drude formula, can be expressed as

$$
\begin{aligned}
\mu(T)=A & {\left[\frac{T}{\Theta_{m}}\right]^{-2} } \\
\times & \left\{J_{2}\left[4 \beta \epsilon_{0}\left[1+\frac{\left(E_{F}\right)^{1 / 2}}{\epsilon_{0}}\right]\right]\right. \\
& \left.-J_{2}\left[4 \beta \epsilon_{0}\left[-1+\frac{\left(E_{F}\right)^{1 / 2}}{\epsilon_{0}}\right]\right]\right\}^{-1},
\end{aligned}
$$

with

$$
A=\frac{\pi N N^{\prime} e m_{i} \hbar^{3} C_{s}^{4}}{m^{*} \xi^{2}\left(k_{B} \Theta_{m}\right)^{2}}
$$

Thus, the temperature dependence of the mobility for a 1D electron gas interacting with an 1D LA-phonon system, described by Eq. (33), is valid over the entire temperature range where the material is metallic.

The temperature dependence of the mobility has been calculated for a $\mathrm{Ga}_{1-x} \mathrm{Al}_{x} \mathrm{As} / \mathrm{GaAs} \mathrm{QWW}$ from $T / \Theta_{m} \simeq 0.1$ to $T=T_{F}, T_{F}$ being temperature which is about $15 \mathrm{~K}$ in this case.

One notes from the log-log plot of Fig. 2 that the theoretical results for the mobility [in units of $A$, cf. Eq. (34)] as a function of the temperature show different power law in $T / \Theta_{m}$ along the temperature range of interest. One observes that in the high-temperature limit, i.e., $T>\Theta_{m}$, the curve has a slope equal to -1 , which is representative of a resisitivity which has a linear increase with $T$, as is expected for $3 \mathrm{D}$ metals. Besides, at low temperatures, i.e., $T<\Theta_{m}$, the negative slope of the curve $\mu / A$ versus $T / \Theta_{m}$ increases rapidly as temperature decreases, from $T^{-2}$ around $T / \Theta_{m} \cong 0.25$ to higher powers 


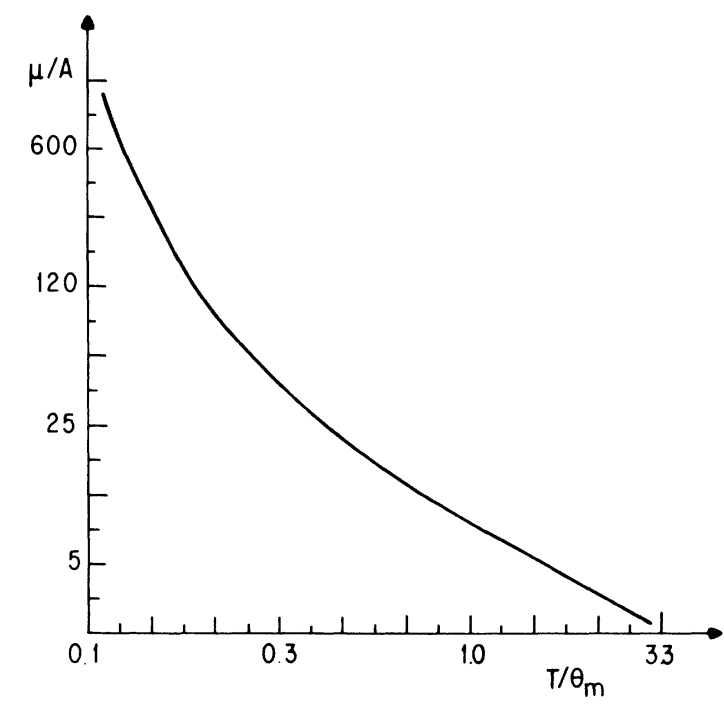

FIG. 2. Temperature dependence of the electrical mobility $\mu(T)$ in units of $A$ at the temperature range $T \ll \Theta_{m}$ and $1<T / \Theta_{m}<T_{F}=15 \mathrm{~K}$ for a GaAs QWW. $A$ is a constant given by Eq. (34).

for very low temperatures.

It is interesting to compare the numerical results obtained in our model of 1D LA-phonon mobility in the entire temperature range with some experimental data and previous calculations to some 1D metals. In particular, Conwell et al. ${ }^{13}$ performed a tight-binding calculation of the resistivity in order to describe the (TMTSF) ${ }_{2} \mathrm{PF}_{6}$ compound, based on the Boltzmann-equation approach, giving rise to a temperature dependence which is proportional to $1 / \sinh \left(\Theta_{m} / T\right)$, with $\Theta_{m}=60 \mathrm{~K}$. In Ref. 13, the calculation is performed in the metallic range, from the Peierls transition temperature $T_{P}=14 \mathrm{~K}$ up to $T \geq \Theta_{m}$.

We notice that the numerical results in our model for this same temperature range, i.e, $T / \Theta_{m} \cong 0.25$ up to $T \geq \Theta_{m}$ show a similar power law in temperature and are in good agreement with the experimental data. Thus, we suggest that using a deformation potential as done in the present work instead of an electron-phonon coupling characteristic of a tight-binding chain as employed in Ref. 13, leads to quite similar results.

\section{FINAL REMARKS}

In this work we have calculated the temperature dependence of the static transport properties of a $1 D$ EG due to scattering by $1 \mathrm{D}$ LA phonons using the memoryfunction formalism. The model is suitable to be applied to materials like $\mathrm{Ga}_{1-x} \mathrm{Al}_{x} \mathrm{As} / \mathrm{GaAs}$ quantum-well-wire systems as well as to $\mathrm{Hg}_{3-\delta} \mathrm{AsF}_{6}$ or (TMTSF) ${ }_{2} \mathrm{PF}_{6}$ systems in the metallic temperature range, above the Peierls transition, taking into account in each case their characteristic $\Theta_{m}$ values.

The theoretical results have shown a complex variety in power law for the temperature dependence of the electrical mobility. At high-temperature limit, i.e., $T \geq \Theta_{m}$, the mobility is inversely proportional to $T$, whereas at low temperatures $\left(T<\Theta_{m}\right)$, the inverse power law in $T$ increases rapidly as temperature decreases, passing from a power law $T^{-2}$ at $T / \Theta_{m} \cong 0.25$ to higher negative power for very low temperatures.

Finally, it is worthwhile to mention that our theory can be generalized in order to obtain dynamic transport properties and to include magnetic field effects on these 1D systems. ${ }^{14}$ Besides, many-body effects can also be introduced in our formalism. In fact, the approximation in Eq. (17), i.e., using "simple bubble" noninteracting electron density correlation $S^{0}\left(q, i p_{n}\right)$ instead of the complete $S\left(\mathrm{q}, i p_{n}\right)$, implies neglecting all "quantum corrections" to the conductivity. However, these effects do not seem to play an important role for the LA-phonon scattering in a simple $1 \mathrm{D}$ metal in the temperature range we are interested in.

\section{ACKNOWLEDGMENT}

I.C.C.L and A. T. want to acknowledge very fruitful discussions with Sankar Das Sarma during the beginning of this work.
*On leave from Instituto de Física, Universidade do Estado do Rio de Janeiro-UERJ, Rio de Janeiro, RJ, Brazil.

${ }^{1}$ R. E. Peierls, Quantum. Theory of Solids (Oxford University Press, New York, 1955), p. 108; H. Frölich, Proc. R. Soc. London, Ser. A 223, 296 (1954).

${ }^{2}$ E. M. Conwell, Phys. Rev. B 22, 1761 (1980).

${ }^{3}$ J. P. Pouget, G. Shirane, J. M. Hastings, A. J. Heeger, N. D. Miro, and A. G. MacDiarmid, Phys. Rev. B 18, 3643 (1978); D. L. Peebles, C. K. Chiang, M. J. Choen, A. J. Heeger, N. D. Miro, and A. G. MacDiarmid, ibid. 15, 4607 (1977).

${ }^{4}$ T. M. Rice, Physics in One-Dimension, proceedings of an international conference, Fribourg, Switzerland, 1980, edited by J. Bernasconi and T. Schneider (Springer-Verlag, Berlin, 1981), p. 229, and references therein.

${ }^{5}$ H. Sakaki, Jpn. J. Appl. Phys. 19, L735 (1980).
${ }^{6}$ P. M. Petroff, A. C. Gossard, R. A. Logan, and W. Wiegman, Appl. Phys. Lett. 41, 635 (1982).

${ }^{7}$ P. A. Lee, Phys. Rev. Lett. 53, 2042 (1984).

${ }^{8}$ S. Das Sarma and We-yan Lai, Phys. Rev. B 32, 1401 (1985).

${ }^{9}$ C. E. Leal, I. C. da Cunha Lima, A. Troper, and S. Das Sarma, Phys. Rev. B 35, 4095 (1987).

${ }^{10}$ W. Götze and P. Wolfe, Phys. Rev. B 6, 1226 (1972).

${ }^{11}$ See, for example, G. D. Mahan, Many Particles Physics (Plenum, New York, 1981).

${ }^{12}$ D. Forster, Hydrodynamic Fluctuations, Broken Symmetry, and Correlation Functions (Benjamin, New York, 1975), p. 95.

${ }^{13}$ E. M. Conwell and C. S. Jacobsen, Solid State Commun. 40, 203 (1981).

${ }^{14}$ K. F. Berggren, G. Roos, and H. van Houten (unpublished). 